Original Article

\title{
Parasitic contamination of fresh vegetables sold in open markets: a public health threat
}

\author{
Contaminação parasítica de vegetais frescos vendidos em mercados abertos: \\ uma ameaça para a saúde pública
}

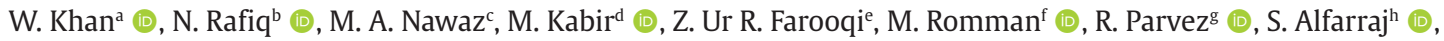 \\ A. Noor ${ }^{\mathrm{i}}$ (D) and A. A. Ujjan ${ }^{\mathrm{j}}$ (D) \\ a University of Malakand, Department of Zoology, Lower Dir, KP, Pakistan \\ ${ }^{\mathrm{b}}$ Abdul Wali Khan University, Department of Zoology, Mardan, KP, Pakistan \\ 'Shaheed Benazir Bhutto University, Department of Biotechnology, Sheringal, Dir (Upper), KPK, Pakistan \\ dUniversity of Sargodha, Department of Biological Sciences, Punjab, Pakistan \\ e University of Karachi, Department of Botany, Karachi, Pakistan \\ ${ }^{\mathrm{f}}$ University of Chitral, Department of Botany, KP, Pakistan \\ ${ }^{g}$ Government Girls Degree College Dargai, Department of Botany, Malakand, KP, Pakistan

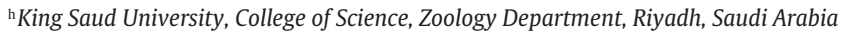 \\ ${ }^{\mathrm{i}}$ Karakoram International University Gilgit, Biological Sciences Department, Baltistan, Pakistan \\ j University of Sindh, Institute of Plant Sciences, Jamshoro, Pakistan
}

\begin{abstract}
Vegetables eating raw are a leading source of transmission of infective forms of pathogenic internal parasites among human beings. This research was conducted from April to October, 2017 to assess the parasitic contamination of vegetables sold at main vegetable markets in districts Lower Dir and Peshawar, Pakistan. Eight hundred specimens of different vegetables were purchased and soaked in physiological saline solution, shaken with a mechanical shaker for 20 minutes and processed by sedimentation concentration method. Results revealed that only $19.7 \%$ ( $n=158 / 800)$ of the vegetables were found to be contaminated with single or multiple parasite species. Ascaris lumbricoides (the large round worm) $12.3 \%(\mathrm{n}=99 / 800)$ was the most commonly detected pathogen and Taenia saginata (the beef tapeworm) $1.62 \%(n=13 / 800)$ was the least frequently detected one. Interestingly, significant $p$ value ( $p>0.05$ at $95 \% \mathrm{CI}$ ) between the number of examined and contaminated for all the variables studied including education status of the vendors, markets location, type of vegetables, means of display, washed before display, washing source of water and market type. The findings of this study evidenced that consumption of raw vegetables possesses great risk of getting parasitic infections in Lower Dir and Peshawar districts, Pakistan. Instructing the sellers and the public about parasitic disease transfer and their hygiene can reduce the infection rate of parasites of human origin.

Keywords: vegetables contamination, intestinal parasites, public health importance, soil-transmitted helminthes, raw vegetables.
\end{abstract}

\begin{abstract}
Resumo
Os vegetais crus são a principal fonte de transmissão de formas infecciosas de parasitas internos patogênicos entre os seres humanos. Esta pesquisa foi realizada de abril a outubro de 2017 para avaliar a contaminação parasitária de vegetais vendidos nos principais mercados de vegetais nos distritos de Lower Dir e Peshawar, Paquistão. Oitocentos exemplares de diferentes vegetais foram adquiridos e embebidos em solução salina fisiológica, agitados em agitador mecânico por 20 minutos e processados pelo método de concentração por sedimentação. Os resultados revelaram que apenas 19,7\% ( $n=158 / 800$ ) das hortaliças estavam contaminadas com uma ou várias espécies de parasitas. Ascaris lumbricoides (o verme redondo grande) $12,3 \%$ ( $\mathrm{n}=99 / 800$ ) foi o patógeno mais comumente detectado e Taenia saginata (a tênia da carne) $1,62 \%(n=13 / 800)$ foi o menos freqüentemente detectado. Curiosamente, valor de p significativo ( $p>0,05$ em IC de 95\%) entre o número de examinados e contaminados para todas as variáveis estudadas incluindo escolaridade dos vendedores, localização dos mercados, tipo de vegetais, meios de exposição, lavado antes da exposição, fonte de lavagem de água e tipo de mercado. Os resultados deste estudo evidenciaram que o consumo de vegetais crus possui grande risco de contrair infecções parasitárias nos distritos de Lower Dir e Peshawar, no Paquistão. Instruir os vendedores e o público sobre a transferência de doenças parasitárias e sua higiene pode reduzir a taxa de infecção de parasitas de origem humana.
\end{abstract}

Palavras-chave: contaminação de vegetais, parasitas intestinais, importância para a saúde pública, helmintos transmitidos pelo solo, vegetais crus.

*e-mail: walikhan.pk@gmail.com

Received: August 23, 2020 - Accepted: October 16, 2020

This is an Open Access article distributed under the terms of the Creative Commons Attribution License, which permits unrestricted use, distribution, and reproduction in any medium, provided the original work is properly cited. 


\section{Introduction}

Fresh vegetables as a potential source of vitamins, dietary fibers and minerals are commonly used in almost all the societies in the world, including Pakistan. Routine consumption of vegetable is related with a minimum risk of cardio vascular diseases, stroke and cancer. Some diseases such as cardio vascular diseases, cancer diabetes, Alzheimer's diseases and cataracts can be minimized by a use of vegetables. Dietitians recommend consuming vegetables daily because of their role in health promotion and disease prevention (Liu, 2003). In spite of the advantages mentioned, the risk of the parasitic infections can be raised by the consumption of vegetables as raw (Maikai et al., 2012)

Uses of fresh vegetables can be directly linked to foodborne illness. Vegetables require a moist environment for their growth, particularly those eaten raw and without peeling, have been demonstrated to be a way for transmission a wide range of parasites (Al-Binali et al., 2006). Various parasites have been associated with vegetables including protozoa cysts and helminth eggs (Daryani et al., 2012). Most of the studies shown the strong association between vegetables especially raw ones and parasitic infections (Ogbolu et al., 2009). The consumption of raw vegetables plays a major epidemiological role in the transmission of parasitic foodborne diseases (Beuchat, 2002). Intestinal parasitic infection may be acquired in different ways like by consumption of contaminated vegetables, other food stuff, and water (Al-Binali et al., 2006). Eating raw, or undercooked vegetables is one of the means by which the transmission of intestinal parasitic infections is propagated (Slifko et al., 2000)

Infective forms of parasites can be transmitted through vegetables, when they are associated with various associated factors as like that of planting, harvesting, transportation, storage, market chain, and even reach to storehouse (Idahosa, 2011). Worldwide 3.5 billion people were have been reported to be infected and 450 million are at risk of intestinal parasitic infections, with an estimated 200,000 deaths annually. (Walked, 2009). Soil transmitted helminthes like $A$. lumbricoides infects over one billion people, Trichuris trichuria 79 million and hookworm (Ancyclostoma duodenale and Necator americanus) 740 million people (WHO, 1987) in the world.

Several reports have been published on intestinal parasitic infection in various groups of humans in the region as Khan et al. (2011, Khan et al., 2012, 2014, 2015, 2016, 2017a, b, c, 2018a, b, 2019a, b, 2020a, b) and Noor-un-Nisa et al. (2012) however, only one report is found published on parasitic contamination of vegetable as Khan et al. (2017c) in the study area. Different types of vegetables in different regions of the world, contaminated with helminth eggs and larvae (Muniswamappa et al., 2012). The parasitic contamination of vegetables depends on factors such as use of untreated wastewater and water supplies contaminated with sewage for irrigation, postharvest handling, and hygienic conditions of preparation in food service and storage setting (Amoah et al., 2007).
Other factors contribute to increase in diseases associated with raw vegetables such as globalization of food supply, introduction of pathogens into new geographical areas through import, use of untreated waste water and manure as fertilizers for crop production, irrigation and various agronomic practices, level of hygiene of food handlers etc. (James and Ogodukwuo, 2006).

This study designates the prevalence of parasitic contamination and their possibility of association with vegetables eaten raw in Chakdara Dir (Lower) and Peshawar districts, Pakistan. Considering the importance of parasitic infection on human health, it is of prime importance to determine whether the available and daily handled and consumed vegetables are contaminated with the parasites or not. And thus the present study was designed.

\section{Materials and Methods}

\subsection{Localities}

This research was conducted from April to October, 2017 to assess the level of parasitic contamination of vegetables sold at main vegetable markets in Lower Dir and Peshawar districts, Pakistan. Lower Dir district lies in the north-west of Khyber Pakhtunkhwa province, Pakistan and covers an area of $1583 \mathrm{~km}^{2}$. Apart from small areas in the south-west, Dir is a rocky, mountainous zone with peaks rising to $5,000 \mathrm{~m}$ in the north-east and to $3,000 \mathrm{~m}$ along the watersheds with Swat to the east and Afghanistan and Chitral to the west and north. According to the census conducted in 2017, the population of Lower Dir is 1,435,917 individuals. Peshawar district is located about $160 \mathrm{~km}$ west of the Pakistan's capital Islamabad. Peshawar district is reserved by 4,269,079 people according to the 2017 census. The mean maximum and minimum temperature during summer is over $40{ }^{\circ} \mathrm{C}$ and $25^{\circ} \mathrm{C}$. The mean maximum and minimum temperature in winter is $18.35{ }^{\circ} \mathrm{C}$ and $4{ }^{\circ} \mathrm{C}$. The rainfall is higher in winter than in the summer. The humidity ranges from $46 \%$ to $76 \%$ in June to August.

\subsection{Data collection}

A questionnaire was designed for collecting information from 63 vendors on causes related with vegetable contamination by parasites such as: educational status of the vendors, main vegetable markets, type of vegetables, means of display, washed before display, sources of water for washing and type of markets.

\subsection{Sample collection and analysis}

Twenty four types of vegetables (L. esculentum (Tomato), C. annum (Chilli), C. sativus (Cucumber), C. annum (green bell pepper), S.melogena (Brinjal), P.sativam (peas), C. vulgaris (Round gourd), L.cylindrica (vegetable sponge), M.charanjtia (bitter gourd), S.tuberosum (potato), Z.officinale (ginger), C. esculenta (taro), A.cepa (onion), A.sativum (garlic), B.rapa (turnip), B.compastris (mustard), A. esculantum (Lady finger), L. sative (Lettuce salad), S.oleracea (spinach), C. annum (coriander), M.longifolia (mint), D.carota (carrot), B.oleracea (Cauliflower), and B.olerace (cabbage) were purchased 
from two main vegetable markets one in each Lower Dir and Peshawar districts.

Each vegetable sample was kept in isolated container and brought to the Zoology laboratory, University of Malakand for parasite contamination. Almost $250 \mathrm{gms}$ of each vegetable was soaked in a half liter of saline solution, shacked with the help of a mechanical shaker for 20 minutes. Kept at night for sedimentation, 10 milliliters of the sediment was taken to a centrifuge tube as to remove undesirable matter. The tube was centrifuged at $3000 \mathrm{rpm}$ for 5 minutes for focused ova, larvae, cysts, and oocysts (Omowaye and Audu, 2012). The supernatant was poured carefully without shaking. The sediment was disturbed by hand to redistribute the parasitic stages. Finally, the sediment was studied under microscope [Labomed 400] using $\times 10$ and $\times 40$ objectives.

\subsection{Statistical analysis}

The data was analyzed by applying the Graph-Pad version-5. P-value was considered significant if less than 0.05 at $95 \%$ confidence interval.

\section{Results}

This study revealed $19.7 \%$ of the vegetable examined was contaminated with any parasitic stage. The percentage of each sample contaminated was given in Table 1: 21 and $25 \%$ of the L. esculentum (Tomato), 15 and $15.3 \%$ C. annum (Chilli), 11.7 and 9.0\% C. sativus (Cucumber), 26 and $29.4 \%$ C. annum (green bell pepper), 15.7 and $30 \%$ S.melogena (Brinjal), 10 and 16.6\% P.sativum (peas), 10 and $15.3 \%$ C. vulgaris (Round gourd), 13.3 and 11.1\% L.cylindrica (vegetable sponge), 23 and 30\% M.charanjtia (bitter gourd), 6.25 and 12.5\% S.tuberosum (potato), 15 and 23.5\% Z.officinale (ginger), 14.2 and $10.5 \%$ C. esculenta (taro), 11.7 and 18.7\% A.cepa (onion), 17.6 and 15.3\% A.sativum (garlic), 20 and $15.7 \%$ B.rapa (turnip), 27.7 and $42.8 \%$ B.compastris (mustard), 22.2 and $21.4 \%$ A. esculantum (Lady finger), 26 and 20\% L. sative (Lettuce salad), 17.3 and $21 \%$ S.oleracea (spinach), 36.8 and $31.2 \%$ C. annum (coriander), 27.7 and 31.2\% M.longifolia (mint), 16.6 and $12.5 \%$ D.carota (carrot), 22.2 and $22.2 \%$ B.oleracea (Cauliflower), 36.3 and 23\% of B.olerace (cabbage) in Lower Dir and Peshawar districts.

Of the examined vegetables more than half (53.7\%) were contaminated with more than one parasite species, including $31.6 \%$ of the samples were contaminated with two species of parasites, $17 \%$ with three species of parasites and $5.06 \%$ were observed contaminated with 4 species of parasites (Table 1 ).

Regarding factors related to the contamination of vegetables interviews were recorded from vendors in the markets. A total of 63 sellers were inquired about their educational status and it was shown that $20 \%$ of the vendors had no proper education, $19.6 \%$ had primary and secondary education (each). Level of education of the sellers and the rate of parasitic contamination of the vegetables were associated significantly $(\mathrm{P}=0.0246)$ (Table 3$)$.

Similar rate of contamination was observed (20.7\%) of the vegetables were contaminated in main vegetable market Peshawar and (18.9\%) vegetables were infected in main vegetable market Chakdara. The association between vegetable collected and that of infected was significant ( $\mathrm{P}=0.0146$, at $\mathrm{CI} 23.62$ to 196.9) (Table 3). Compared to type of vegetable collected $C$. annum (coriander) was highly infected while S.tuberosum (potato) showed least (9.37\%) of the parasitic contamination. This association was statistically significant ( $\mathrm{P}=0.0001, \mathrm{Cl} 23.45$ to 30.05 ), regarding the factor means of display of the vegetables ( $\mathrm{P}=0.0059, \mathrm{CI} 32.14$ to 173.7), wash before display 0.0216 , 17.50 to $203.1 \mathrm{CI}$ ); Source of water for washing ( $\mathrm{P}=0.0115$, 24.95 to $180.9 \mathrm{CI}$ ) (Table 3 ).

\section{Discussion}

People in developing countries are mostly infected with intestinal parasites and raw vegetable used is one of the important route of their transmission. Eight hundred vegetable samples of 24 different types from the main markets in districts Lower Dir and Peshawar were examined. Only $19.7 \%$ were positive for intestinal parasitic contamination. Recently, Khan et al. (2017c) examined 520 samples of thirteen different types of fresh vegetables from two main vegetable markets in lower Dir and Upper Dir districts in Khyber Pakhtunkhwa, Pakistan, of which $10.7 \%$ were positive for helminth eggs and Entamoeba histolytica cysts. Dias et al. (2014) found intestinal parasites in $42 \%$ in freshly leafy vegetables consumed in Manglore. Inclusion of highly contaminated fresh vegetables in routine foods will expose consumers to the risk of acquiring intestinal parasites.

In present study the overall parasitic contamination of vegetables were $19.7 \%$. Current research agreed with the findings conducted in Khartoum, state, Sudan 13.5\% (Mohamed et al., 2016) and Lower and Upper Dir districts 10.7\% (Khan et al., 2017c) and in Tehran, Iran 8.5\% (Valipour , 2015); In other studies higher number of vegetables were found contaminated with human intestinal parasites such as Jimma Town, Southwest Ethiopia 58.7\% (Tefera et al., 2014); Benha, Egypt 29.6\% (Eraky et al., 2014); Amol, North of Iran 46.5\% (Siyadatpanah et al., 2013); Zahedan, Iran 44.8\% (Ebrahimzadeh et al., 2013); Manglore 42\% (Dias et al., 2014); Iran 48.4\% (Mehrnejat et al., 2015); Lahore, Pakistan 31.2\% (Maqbool et al., 2014); Ebonyi state, Nigeria 60\%; Kurramabad, Iran 52.7\% (Ezatpour et al., 2013) and Ebonyi state, Nigeria 55\% (Lyabo and Oluchi, 2015).

In present study, $12.3 \%$ of the fresh vegetables were found contaminated with Ascaris lumbricoides eggs, the most common parasite detected. Findings of the present research agrees with the studies conducted in Zahedan Iran 6.1\% (Ebrahimzadeh et al., 2013); Jimmu town Southeast Ethiopia 6.7\% (Tefera et al., 2014); Kogi state, Nigeria 5.6\% and Khurram abad, Iran 4.7\% (Ezatpour et al., 2013). Ascaris lumbricoides was reported in higher prevalence in studies carried out in different parts of the world by different workers as Tabuk, Saudi Arabia 21.7\% (Gabre and Shakir, 2016); Nigeria 54.5\% (Elom et al., 2012); Manglore 23\% (Dias et al., 2014); Lahore, Pakistan 37.1\% (Maqbool et al., 2014); Eastern Showa, Ethiopia 
Table 1. Pattern of parasitological contamination of vegetables in selected markets in districts Lower Dir (LD) and Peshawar (PR) (April to October, 2017).

\begin{tabular}{|c|c|c|c|c|c|c|c|}
\hline \multirow{2}{*}{ Type of vegetable } & \multirow{2}{*}{ Locality } & \multirow{2}{*}{$\begin{array}{l}\text { No. } \\
\text { examined }\end{array}$} & \multirow{2}{*}{$\begin{array}{c}\text { No. } \\
\text { positive } \\
(\%)\end{array}$} & \multicolumn{4}{|c|}{ No.of parasite species detected (\%) } \\
\hline & & & & single & double & triple & quadruple \\
\hline \multirow{2}{*}{$\begin{array}{l}\text { L. esculentum } \\
\text { (Tomato) }\end{array}$} & LD & 19 & $4(21)$ & $2(10.5)$ & $1(5.26)$ & $1(5.26)$ & 0 \\
\hline & PR & 12 & $3(25)$ & $2(16.6)$ & $1(8.33)$ & 0 & 0 \\
\hline \multirow{2}{*}{ C. annum (Chilli) } & LD & 20 & $3(15)$ & $2(10)$ & $1(5)$ & 0 & 0 \\
\hline & PR & 13 & $2(15.3)$ & $2(15.3)$ & 0 & 0 & 0 \\
\hline \multirow{2}{*}{$\begin{array}{l}\text { C. sativus } \\
\text { (Cucumber) }\end{array}$} & LD & 17 & $2(11.7)$ & $1(5.88)$ & $1(5.88)$ & 0 & 0 \\
\hline & PR & 11 & $1(9.0)$ & $1(9.0)$ & 0 & 0 & 0 \\
\hline \multirow{2}{*}{$\begin{array}{l}\text { C. annum (green } \\
\text { bell pepper) }\end{array}$} & LD & 23 & $6(26.0)$ & $3(13.0)$ & $2(8.69)$ & $1(4.34)$ & 0 \\
\hline & PR & 17 & $5(29.4)$ & $2(11.7)$ & $1(5.88)$ & $1(5.88)$ & $1(5.88)$ \\
\hline \multirow{2}{*}{ S.melogena (Brinjal) } & LD & 19 & $3(15.7)$ & $2(10.5)$ & $1(5.26)$ & 0 & 0 \\
\hline & PR & 10 & $3(30)$ & $2(20)$ & $1(10)$ & 0 & 0 \\
\hline \multirow{2}{*}{ P.sativam (peas) } & LD & 30 & $3(10)$ & $1(3.33)$ & $1(3.33)$ & $1(3.33)$ & 0 \\
\hline & PR & 24 & $4(16.6)$ & $2(8.30)$ & $1(4.16)$ & $1(4.16)$ & 0 \\
\hline \multirow{2}{*}{$\begin{array}{l}\text { C. vulgaris (Round } \\
\text { gourd) }\end{array}$} & LD & 20 & $2(10)$ & $1(5)$ & $1(5)$ & 0 & 0 \\
\hline & PR & 13 & $2(15.3)$ & $1(7.69)$ & $1(7.69)$ & 0 & 0 \\
\hline \multirow{2}{*}{$\begin{array}{l}\text { L.cylindrica } \\
\text { (vegetable sponge) }\end{array}$} & LD & 15 & $2(13.3)$ & 0 & $2(13.3)$ & 0 & 0 \\
\hline & PR & 09 & $1(11.1)$ & $1(11.1)$ & 0 & 0 & 0 \\
\hline \multirow{2}{*}{$\begin{array}{l}\text { M.charanjtia (bitter } \\
\text { gourd) }\end{array}$} & LD & 13 & $3(23.0)$ & $1(7.69)$ & $2(15.3)$ & 0 & 0 \\
\hline & PR & 10 & $3(30)$ & $2(20)$ & $1(10)$ & 0 & 0 \\
\hline \multirow{2}{*}{$\begin{array}{l}\text { S.tuberosum } \\
\text { (potato) }\end{array}$} & LD & 16 & $1(6.25)$ & $1(6.25)$ & 0 & 0 & 0 \\
\hline & PR & 16 & $2(12.5)$ & 0 & $2(12.5)$ & 0 & 0 \\
\hline \multirow{2}{*}{ Z.officinale (ginger) } & LD & 20 & $3(15)$ & $1(5)$ & $1(5)$ & $1(5)$ & 0 \\
\hline & PR & 17 & $4(23.5)$ & $2(11.7)$ & $1(5.88)$ & $1(5.88)$ & 0 \\
\hline \multirow{2}{*}{ C. esculenta (taro) } & LD & 21 & $3(14.2)$ & $1(4.76)$ & $1(4.76)$ & $1(4.76)$ & 0 \\
\hline & PR & 19 & $2(10.5)$ & $1(5.26)$ & $1(5.26)$ & 0 & 0 \\
\hline \multirow{2}{*}{ A.cepa (onion) } & LD & 17 & $2(11.7)$ & $1(5.88)$ & 0 & $1(5.88)$ & 0 \\
\hline & PR & 16 & $3(18.7)$ & $1(6.25)$ & $2(12.5)$ & 0 & 0 \\
\hline \multirow{2}{*}{ A.sativum (garlic) } & LD & 17 & $3(17.6)$ & $2(11.7)$ & $1(5.88)$ & 0 & 0 \\
\hline & PR & 13 & $2(15.3)$ & $1(7.69)$ & $1(7.69)$ & 0 & 0 \\
\hline \multirow{2}{*}{ B.rapa (turnip) } & LD & 20 & $4(20)$ & $2(10)$ & $1(5)$ & $1(5)$ & 0 \\
\hline & PR & 19 & $3(15.7)$ & $1(5.26)$ & $1(5.26)$ & 0 & 0 \\
\hline \multirow{2}{*}{$\begin{array}{l}\text { B.compastris } \\
\text { (mustard) }\end{array}$} & LD & 18 & $5(27.7)$ & $2(11.1)$ & $1(11.1)$ & $1(11.1)$ & $1(11.1)$ \\
\hline & PR & 14 & $6(42.8)$ & $3(21.4)$ & $1(7.14)$ & $2(14.2)$ & 0 \\
\hline \multirow{2}{*}{$\begin{array}{l}\text { A. esculantum (Lady } \\
\text { finger) }\end{array}$} & LD & 18 & $4(22.2)$ & $1(5.55)$ & $1(5.55)$ & $2(11.1)$ & 0 \\
\hline & PR & 14 & $3(21.4)$ & $2(14.2)$ & $2(14.2)$ & 0 & 0 \\
\hline \multirow{2}{*}{$\begin{array}{l}\text { L. sative (Lettuce } \\
\text { salad) }\end{array}$} & LD & 23 & $6(26)$ & $2(8.69)$ & $1(4.34)$ & $2(8.69)$ & $1(4.34)$ \\
\hline & PR & 20 & $4(20)$ & $2(10)$ & $1(5)$ & $1(5)$ & 0 \\
\hline \multirow{2}{*}{ S.oleracea (spinach) } & LD & 23 & $4(17.3)$ & $2(8.69)$ & 1(4.340 & $1(4.34)$ & 0 \\
\hline & PR & 19 & $4(21)$ & $1(5.26)$ & 0 & $2(10.5)$ & $1(5.26)$ \\
\hline
\end{tabular}

The reported parasite species include ova of A. lumbricoides, A.duodenale, S.stercoralis, H.nana, T.trichura, E.histolytica and T.saginata, E.vermicularis, F.hepatica and G.lamblia cysts (each). A. lumbricoides (6.62\%) was the most commonly detected parasite, followed by A.duodenale (4.25\%), S.stercoralis (3.37\%), H.nana (2.50\%), T.trichura (1.75\%), E.histolytica (1.25\%), T.saginata $0.25 \%$ (Table 2). 
Table 1. Continued...

\begin{tabular}{|c|c|c|c|c|c|c|c|}
\hline \multirow{2}{*}{ Type of vegetable } & \multirow{2}{*}{ Locality } & \multirow{2}{*}{$\begin{array}{c}\text { No. } \\
\text { examined }\end{array}$} & \multirow{2}{*}{$\begin{array}{c}\text { No. } \\
\text { positive } \\
(\%)\end{array}$} & \multicolumn{4}{|c|}{ No.of parasite species detected (\%) } \\
\hline & & & & single & double & triple & quadruple \\
\hline \multirow{2}{*}{$\begin{array}{l}\text { C. annum } \\
\text { (coriander) }\end{array}$} & LD & 19 & $7(36.8)$ & $2(10.5)$ & $2(10.5)$ & $1(5.26)$ & $2(10.5)$ \\
\hline & PR & 16 & $5(31.2)$ & $1(6.25)$ & $2(12.5)$ & $2(12.5)$ & 0 \\
\hline \multirow{2}{*}{ M.longifolia (mint) } & LD & 18 & $5(27.7)$ & $2(11.1)$ & $1(5.55)$ & $1(5.55)$ & $1(5.55)$ \\
\hline & PR & 16 & $5(31.2)$ & $2(12.5)$ & 0 & $2(12.5)$ & $1(6.25)$ \\
\hline \multirow{2}{*}{ D.carota (carrot) } & LD & 18 & $3(16.6)$ & $2(11.1)$ & $1(5.55)$ & 0 & 0 \\
\hline & PR & 16 & $2(12.5)$ & $1(6.25)$ & $1(6.25)$ & 0 & 0 \\
\hline \multirow{2}{*}{$\begin{array}{l}\text { B.oleracea } \\
\text { (Cauliflower) }\end{array}$} & LD & 09 & $2(22.2)$ & $2(22.2)$ & 0 & 0 & 0 \\
\hline & PR & 09 & $2(22.2)$ & $1(11.1)$ & $1(11.1)$ & 0 & 0 \\
\hline \multirow{2}{*}{ B.olerace (cabbage) } & LD & 11 & $4(36.3)$ & $2(18.1)$ & $2(18.1)$ & 0 & 0 \\
\hline & PR & 13 & $3(23)$ & $1(7.69)$ & $2(15.3)$ & 0 & 0 \\
\hline Total & & 800 & $158(19.7)$ & $73(46.2)$ & $50(31.6)$ & $27(17)$ & $8(5.06)$ \\
\hline
\end{tabular}

The reported parasite species include ova of A. lumbricoides, A.duodenale, S.stercoralis, H.nana, T.trichura, E.histolytica and T.saginata, E.vermicularis, F.hepatica and G.lamblia cysts (each). A. lumbricoides (6.62\%) was the most commonly detected parasite, followed by A.duodenale (4.25\%), S.stercoralis (3.37\%), H.nana (2.50\%), T.trichura (1.75\%), E.histolytica (1.25\%), T.saginata $0.25 \%$ (Table 2).

Table 2. Parasite species in relation to the type of fresh vegetables collected from both the markets in districts Lower Dir (LD) and Peshawar (PR) April to October, 2017.

\begin{tabular}{|c|c|c|c|c|c|c|c|c|c|c|c|}
\hline Vegetables & 矛 & 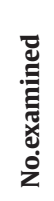 & 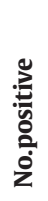 & 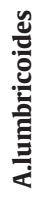 & 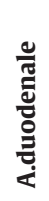 & 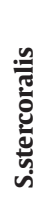 & $\underset{\mathfrak{I}}{\stackrel{\Xi}{\Xi}}$ & 莺 & 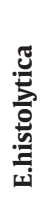 & 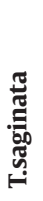 & Total (\%) \\
\hline \multirow{2}{*}{$\begin{array}{l}\text { L. esculentum } \\
\text { (tomato) }\end{array}$} & LD & 19 & 4 & 3 & 2 & 2 & 0 & 0 & 0 & 0 & $7(36.8)$ \\
\hline & PR & 12 & 3 & 2 & 0 & 1 & 1 & 0 & 0 & 0 & $4(33.3)$ \\
\hline \multirow[t]{2}{*}{ C. annum (chilli) } & LD & 20 & 3 & 2 & 1 & 0 & 1 & 0 & 0 & 0 & $4(20)$ \\
\hline & PR & 13 & 2 & 1 & 1 & 1 & 0 & 0 & 1 & 0 & $4(30.7)$ \\
\hline \multirow[t]{2}{*}{ C. sativus (cucumber) } & $\mathrm{LD}$ & 17 & 2 & 2 & 0 & 0 & 0 & 1 & 0 & 0 & $3(17.6)$ \\
\hline & PR & 11 & 1 & 1 & 0 & 0 & 0 & 0 & 0 & 0 & $1(9.09)$ \\
\hline \multirow{2}{*}{$\begin{array}{l}\text { C. annum (green bell } \\
\text { pepper) }\end{array}$} & LD & 23 & 6 & 4 & 2 & 2 & 1 & 0 & 0 & 1 & $10(43.4)$ \\
\hline & PR & 17 & 5 & 4 & 0 & 3 & 0 & 2 & 2 & 0 & 11(64.7) \\
\hline \multirow[t]{2}{*}{ S.melogena (brinjal) } & $\mathrm{LD}$ & 19 & 3 & 2 & 0 & 1 & 0 & 1 & 0 & 0 & $4(21.0)$ \\
\hline & PR & 10 & 3 & 0 & 2 & 0 & 0 & 0 & 1 & 1 & $4(40)$ \\
\hline \multirow[t]{2}{*}{ P.sativam (peas) } & LD & 30 & 3 & 4 & 2 & 0 & 0 & 0 & 0 & 0 & $6(20)$ \\
\hline & PR & 24 & 4 & 4 & 0 & 2 & 2 & 0 & 0 & 0 & $7(29.1)$ \\
\hline \multirow{2}{*}{$\begin{array}{l}\text { C. vulgaris (round } \\
\text { gourd) }\end{array}$} & LD & 20 & 2 & 2 & 1 & 0 & 0 & 0 & 0 & 0 & $3(15)$ \\
\hline & PR & 13 & 2 & 0 & 2 & 0 & 0 & 0 & 1 & 0 & $3(23.0)$ \\
\hline \multirow{2}{*}{$\begin{array}{l}\text { L.cylindrica (vegetable } \\
\text { sponge) }\end{array}$} & LD & 15 & 2 & 0 & 2 & 2 & 0 & 0 & 0 & 0 & $4(26.6)$ \\
\hline & PR & 09 & 1 & 1 & 0 & 0 & 0 & 0 & 0 & 0 & $1(11.1)$ \\
\hline \multirow{2}{*}{$\begin{array}{l}\text { M.charanjtia (bitter } \\
\text { gourd) }\end{array}$} & LD & 13 & 3 & 3 & 2 & 0 & 0 & 0 & 0 & 0 & $5(38.4)$ \\
\hline & PR & 10 & 3 & 0 & 2 & 1 & 1 & 0 & 0 & 0 & $4(40)$ \\
\hline \multirow[t]{2}{*}{ S.tuberosum (potato) } & LD & 16 & 1 & 1 & 0 & 0 & 0 & 0 & 0 & 0 & $1(6.25)$ \\
\hline & PR & 16 & 2 & 0 & 2 & 0 & 0 & 2 & 0 & 0 & $4(25)$ \\
\hline \multirow[t]{2}{*}{ Z.officinale (ginger) } & $\mathrm{LD}$ & 20 & 3 & 2 & 2 & 0 & 0 & 2 & 0 & 0 & $6(30)$ \\
\hline & PR & 17 & 4 & 4 & 2 & 0 & 1 & 0 & 0 & 0 & $7(41.1)$ \\
\hline
\end{tabular}


Table 2. Continued...

\begin{tabular}{|c|c|c|c|c|c|c|c|c|c|c|c|}
\hline Vegetables & : & 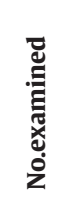 & 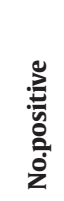 & 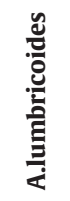 & 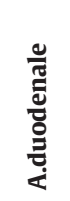 & 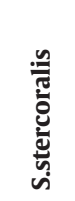 & $\stackrel{\overparen{\Xi}}{\stackrel{\Xi}{\Xi}}$ & 芯 & 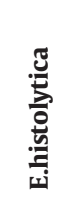 & 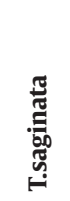 & Total (\%) \\
\hline \multirow[t]{2}{*}{ C. esculenta (taro) } & LD & 21 & 3 & 2 & 0 & 2 & 2 & 0 & 0 & 0 & $6(28.5)$ \\
\hline & PR & 19 & 2 & 2 & 1 & 0 & 0 & 0 & 0 & 0 & $3(15.7)$ \\
\hline \multirow[t]{2}{*}{ A.cepa (onion) } & LD & 17 & 2 & 0 & 0 & 0 & 0 & 0 & 2 & 2 & $4(23.5)$ \\
\hline & PR & 16 & 3 & 1 & 1 & 0 & 0 & 1 & 0 & 0 & $3(18.7)$ \\
\hline \multirow[t]{2}{*}{ A.sativum (garlic) } & LD & 17 & 3 & 2 & 1 & 1 & 0 & 0 & 0 & 0 & $4(23.5)$ \\
\hline & PR & 13 & 2 & 0 & 0 & 2 & 1 & 0 & 0 & 0 & $3(23.0)$ \\
\hline \multirow[t]{2}{*}{ B.rapa (turnip) } & LD & 20 & 4 & 4 & 2 & 1 & 0 & 0 & 0 & 0 & $7(35)$ \\
\hline & PR & 19 & 3 & 0 & 0 & 0 & 0 & 1 & 1 & 1 & $3(15.7)$ \\
\hline \multirow{2}{*}{$\begin{array}{l}\text { B.compastris } \\
\text { (mustard) }\end{array}$} & LD & 18 & 5 & 5 & 2 & 0 & 0 & 2 & 2 & 0 & 11(61.1) \\
\hline & PR & 14 & 6 & 6 & 2 & 1 & 1 & 1 & 0 & 0 & $11(78.5)$ \\
\hline \multirow{2}{*}{$\begin{array}{l}\text { A. esculantum (lady } \\
\text { finger) }\end{array}$} & LD & 18 & 4 & 3 & 2 & 2 & 0 & 0 & 0 & 2 & $9(50)$ \\
\hline & PR & 14 & 3 & 0 & 2 & 2 & 0 & 0 & 2 & 0 & $6(42.8)$ \\
\hline \multirow{2}{*}{$\begin{array}{l}\text { L. sative (lettuce } \\
\text { salad) }\end{array}$} & LD & 23 & 6 & 5 & 3 & 3 & 3 & 0 & 0 & 0 & $14(60.8)$ \\
\hline & PR & 20 & 4 & 2 & 2 & 0 & 2 & 0 & 1 & 0 & $7(35)$ \\
\hline \multirow[t]{2}{*}{ S.oleracea (spinach) } & LD & 23 & 4 & 0 & 0 & 0 & 0 & 2 & 2 & 3 & $7(30.4)$ \\
\hline & PR & 19 & 4 & 4 & 3 & 2 & 0 & 2 & 0 & 0 & $11(57.8)$ \\
\hline \multirow[t]{2}{*}{ C. annum (coriander) } & LD & 19 & 7 & 6 & 3 & 3 & 2 & 0 & 3 & 0 & $17(89.4)$ \\
\hline & PR & 16 & 5 & 3 & 3 & 0 & 2 & 2 & 0 & 1 & $11(68.7)$ \\
\hline \multirow[t]{2}{*}{ M.longifolia (mint) } & LD & 18 & 5 & 4 & 3 & 2 & 2 & 0 & 0 & 0 & 11(61.1) \\
\hline & PR & 16 & 5 & 5 & 0 & 2 & 0 & 3 & 2 & 0 & $12(75)$ \\
\hline \multirow[t]{2}{*}{ D.carota (carrot) } & LD & 18 & 3 & 0 & 0 & 0 & 0 & 2 & 1 & 1 & $4(22.2)$ \\
\hline & PR & 16 & 2 & 0 & 0 & 2 & 1 & 0 & 0 & 0 & $3(18.7)$ \\
\hline \multirow{2}{*}{$\begin{array}{l}\text { B.oleracea } \\
\text { (cauliflower) }\end{array}$} & LD & 09 & 2 & 1 & 1 & 0 & 0 & 0 & 0 & 0 & $2(22.2)$ \\
\hline & PR & 09 & 2 & 0 & 2 & 0 & 1 & 0 & 0 & 0 & $3(33.3)$ \\
\hline \multirow[t]{2}{*}{ B.olerace (cabbage) } & LD & 11 & 4 & 2 & 2 & 0 & 1 & 0 & 1 & 0 & $6(54.5)$ \\
\hline & PR & 13 & 3 & 0 & 2 & 2 & 0 & 0 & 0 & 1 & $5(38.4)$ \\
\hline Total & & 800 & 158 & 99 & 62 & 42 & 25 & 23 & 22 & 13 & 286 \\
\hline Percent (\%) & & & & 12.3 & 7.75 & 5.25 & 3.12 & 2.87 & 2.75 & 1.62 & \\
\hline
\end{tabular}

Table 3. Factors affecting on the contamination of vegetables sold in local markets in districts Lower Dir and Peshawar (April to October, 2017).

\begin{tabular}{lcccc}
\hline \multicolumn{1}{c}{ Variable } & \multicolumn{4}{c}{ Results of parasitological analysis } \\
\cline { 2 - 5 } & Total & Positive (\%) & 95\% Confidence of interval & P value \\
\hline Educational status of vendors & & & 44.88 to 383.1 & 0.0246 \\
• No education & 170 & $34(20)$ & & \\
• Primary education & 376 & $54(19.6)$ & & \\
• Secondary education & 254 & $50((19.6)$ & & \\
Total & 800 & & & \\
Markets & & 848.0146 & \\
• Main vegetable market Chakdara & 444 & & & \\
\hline
\end{tabular}


Table 2. Continued.

\begin{tabular}{|c|c|c|c|c|}
\hline \multirow{2}{*}{ Variable } & \multicolumn{4}{|c|}{ Results of parasitological analysis } \\
\hline & Total & Positive (\%) & 95\% Confidence of interval & P value \\
\hline - Main vegetable market Peshawar & 356 & $74(20.7)$ & & \\
\hline Total & 800 & 158 & & \\
\hline \multicolumn{5}{|l|}{ Vegetable } \\
\hline • L. esculentum (Tomato) & 31 & $7(22.5)$ & 23.45 to 30.05 & $<0.0001$ \\
\hline - C. annum (Chilli) & 32 & $5(15.6)$ & & \\
\hline • C. sativus (Cucumber) & 28 & $3(10.7)$ & & \\
\hline - C. annum (green bell pepper) & 40 & $11(27.5)$ & & \\
\hline - S.melogena (Brinjal) & 29 & $6(20.6)$ & & \\
\hline - P.sativam (peas) & 54 & $7(12.9)$ & & \\
\hline - C. vulgaris (Round gourd) & 33 & $4(12.1)$ & & \\
\hline - L.cylindrica (vegetable sponge) & 24 & $3(12.5)$ & & \\
\hline - M.charanjtia (bitter gourd) & 23 & $6(26.0)$ & & \\
\hline - S.tuberosum (potato) & 32 & $3(9.37)$ & & \\
\hline •Z.officinale (ginger) & 37 & $7(18.9)$ & & \\
\hline - C. esculenta (taro) & 40 & $5(12.5)$ & & \\
\hline - A.cepa (onion) & 33 & $5(12.1)$ & & \\
\hline - A.sativum (garlic) & 30 & $5(16.6)$ & & \\
\hline - B.rapa (turnip) & 39 & $7(17.9)$ & & \\
\hline - B.compastris (mustard) & 33 & $11(33.3)$ & & \\
\hline - A. esculantum (Lady finger) & 32 & $7(21.8)$ & & \\
\hline - L. sative (Lettuce salad) & 43 & $10(23.2)$ & & \\
\hline - S.oleracea (spinach) & 42 & $8(19.0)$ & & \\
\hline - C. annum (coriander) & 35 & $12(34.2)$ & & \\
\hline • M.longifolia (mint) & 34 & $10(29.4)$ & & \\
\hline - D.carota (carrot) & 34 & $5(14.7)$ & & \\
\hline - B.oleracea (Cauliflower) & 18 & $4(22.2)$ & & \\
\hline - B.olerace (cabbage) & 24 & $7(29.1)$ & & \\
\hline Total & 800 & 158 & & \\
\hline \multicolumn{5}{|l|}{ Means of display } \\
\hline - On the floor & 389 & 77(19.7) & 32.14 to 173.7 & 0.0059 \\
\hline - On the top of tables & 185 & $36(19.4)$ & & \\
\hline - On the wheel barrow & 226 & $45(19.9)$ & & \\
\hline Total & 800 & 158 & & \\
\hline \multicolumn{5}{|l|}{ Washed before display } \\
\hline • Yes & 557 & $110(19.7)$ & 17.50 to 203.1 & 0.0216 \\
\hline • No & 243 & $48(19.7)$ & & \\
\hline Total & 800 & 158 & & \\
\hline \multicolumn{5}{|l|}{ Source of water for washing } \\
\hline - Pipe & 483 & $95(19.6)$ & 24.95 to 180.9 & 0.0115 \\
\hline • River & 207 & $41(19.8)$ & & \\
\hline •Well & 110 & $22(20)$ & & \\
\hline Total & 800 & 158 & & \\
\hline \multicolumn{5}{|l|}{ Market type } \\
\hline - Grocery & 238 & $47(19.7)$ & 17.09 to $203.5^{+}$ & 0.0222 \\
\hline - Open market & 562 & 111(19.7) & & \\
\hline Total & 800 & 158 & & \\
\hline
\end{tabular}


22.2\% (Benti and Gemechu 2014); Ebonyi state, Nigeria 20.4\%; Lower and Upper Dir districts, Pakistan 26.7\% (Khan et al., 2017); Tripoli-Libya 68\% (' et al., 2009) and Nigeria 23.8\% (Lyabo and Oluchi, 2015). Some low rate of prevalence was noted in the studies conducted in Benha Egypt 0.6\% (Eraky et al., 2014); Tukkey 2.0\% (Avcioğlu et al., 2011) and Khrtoum state, Sudan 2.9\% (Mohamed et al., 2016).

Hook worms prevalence rate was $7.75 \%$ in present study, which was comparable with the findings of Kogi state, Nigeria 5.3\% and Khartoum, state, Sudan 5.7\% (Mohamed et al., 2016). This nematode was found to contaminated the vegetables in higher prevalence rate as Abakaliki Nigeria, 23.8\% (Elom et al., 2102); Manglore 9.52\% (Dias et al., 2014); Accra, Ghana 13\% Duedu et al. (2014); Lahore, Pakistan 10.8\% (Maqbool et al., 2014); Ebonyi state, Nigeria 24.8\% and Ebonyi state, Nigeria 33.3\% (Lyabo and Oluchi, 2015).

Strongyloides stercoralis $5.25 \%$ was the third most prevalent parasite detected in the present investigation. Findings of the present study was comparable with the findings of the research conducted in Eastern Showa, Ethiopia 6.2\% (Benti and Gemechu, 2014). However, in other studies higher contamination rate for this nematode was reported as Jimmu town Southwest Ethiopia 21.9\% (Tefera et al., 2014); Ebonyi state Southeast Nigeria 6.9\% (Elom et al., 2012); Manglore 7.14\% larvae (Dias et al., 2014); Accra, Ghana 43\% Duedu et al., 2014); Tabuk, Saudi Arabia 9.24\% (Gabre and Shakir, 2016); Lahore, Pakistan 8.97\% (Maqbool et al., 2014); Ebonyi state, Nigeria 28.3\%; Ebonyi state, Nigeria 22.2\% (Lyabo and Oluchi, 2015); Khartoum, state, Sudan $8.7 \%$ (Mohamed et al., 2016). This nematode was reported in low rate of prevalence in Zahidan Iran 1\%(Ebrahimzadeh et al., 2013); Kurramabad, Iran 1.1\% (Ezatpour et al., 2013).

Of the total vegetables screened for human intestinal parasitic infection only $3.12 \%$ was contaminated by Hymenolepis nana. Some of the study's findings were found comparable such as Amol, North of Iran 2.1\% (Siyadatpanah et al., 2013); Zahidan Iran 5\% (Ebrahimzadeh et al., 2013); Tabuk, Saudi Arabia 2.7\% (Gabre and Shakir, 2016) and Lahore, Pakistan 5.76\% (Maqbool et al., 2014).Higher contamination rate was reported in Jimmu town Southwest Ethiopia 8.3\% (Tefera et al., 2014) and Benha, Egypt 100\% (Eraky et al., 2014).

Contamination rate of Trichuris trichura was $2.87 \%$ in the present research, which can be compare with studies reported in Zahidan Iran 1\% (Ebrahimzadeh et al., 2013); Accra, Ghana 2\% (Duedu et al., 2014); Kogi state Nigeria 1.4\% and Khartoum, state, Sudan 2.9\% (Mohamed et al., 2016). Some higher contamination rate was found in Ebonyi state Southeast Nigeria 8.90\% (Elom et al., 2012); Manglore 9.52\% larvae (Dias et al., 2014); Lahore, Pakistan 6.41\% (Maqbool et al., 2014); Ebonyi state, Nigeria 18.6\%; Lower and Upper Dir districts 19.6\% (Khan et al., 2017c) and Ebonyi state, Nigeria 9.52\% (Lyabo and Oluchi, 2015). This nematode is variable in prevalence and has a wide geographic distribution.

Entamoeba histolytica was found in $2.75 \%$ of the vegetable examined. This is the lowest rate of vegetable contamination, in all other studies the rate of vegetable contamination was higher with this nematode such as studies in Jimmu town Southwest Ethiopia 5.3\% (Tefera et al., 2014); Benha Egypt 100\% (Eraky et al., 2014); Amol, North of Iran 3.2\% (Siyadatpanah et al., 2013); Zahidan Iran 5\% (Ebrahimzadeh et al., 2013); Accra, Ghana 7\% (Duedu et al., 2014); Tabuk, Saudi Arabia 23.9\% (Gabre and Shakir, 2016); Lahore, Pakistan 7.05\% (Maqbool et al., 2014); Eastern Showa, Ethiopia 12.5\% (Benti and Gemechu, 2014); Ebonyi state, Nigeria 20.7\%; Lower and Upper Dir districts 28.5\% (Khan et al., 2017c); Khartoum, state, Sudan 42.9\% (Mohamed et al., 2016).

Contamination of vegetables found contaminated with Taenia saginata $1.62 \%$ was the least one. Studies conducted in Amol, North of Iran 3.2\% (Siyadatpanah et al., 2013); Zahidan Iran 13.2\% (Ebrahimzadeh et al., 2013); Manglore 11.9\% (Dias et al., 2014); Tabuk, Saudi Arabia 2.17\% (Gabre and Shakir, 2016); Lahore, Pakistan 5.12\% (Maqbool et al., 2014) showed the higher rate of contamination as compared to the present study findings.

Present study showed only 9.12\% ( $n=73 / 800)$ of the vegetables were found contaminated with single parasitism while $10.6 \%(n=85 / 800)$ with multiple parasites species. Bekele et al. (2017) reported $45.6 \%$ ( $n=164 / 360)$ of the vegetables screened for parasitic contamination with single while $8.88 \%(n=32 / 360)$ with more than one species of parasites. The multiple parasite contamination in vegetables used routinely is needed to be studied thoroughly.

Highest and lowest rate of parasitic contamination found in different vegetables screened in different parts of the world by different workers as coriander and potato 89.4\% and 6.25\% Lower Dir district (present study); mint and cucumber 75\% and 9.09\% Peshawar district (present study); coriander and ginger $14.2 \%$ and $1.78 \%$ Lower and Upper Dir districts, Pakistan (Khan et al., 2017c); leek and green onion $80 \%$ and $34.5 \%$ Khoramabad, Iran (Ezatpour et al., 2013); lettuce and cucumber 28.6\% and $11.1 \%$ Ebonyi State, Nigeria; spinach and cabbage highest and lowest Eastern showa, Ethiopia (Benti and Gemechu, 2014); lettuce and chilli 48\% and 16\% Lahore, Pakistan (Maqbool et al., 2014); tomato and okro $20.9 \%$ and $10.2 \%$ in Kogi State, Nigeria;cucumber and tomato $15.7 \%$ and $2.72 \%$ Tabuk, Saudi Arabia (Gabre and Shakir, 2016);lettuce and cabbage 61\% and 18\% Accra, Ghana (Duedu et al., 2014); lettuce and leek 45.5\% and 10.5\% Benha, Egypt (Eraky et al., 2014); spinach and coriander $17.2 \%$ and $3.2 \%$ Amol, North of Iran (Siyadatpanah et al., 2013) respectively.

\section{Conclusion}

Findings of this study disclose that parasitic contaminated vegetables poses health hazards to the consumers if consumed without proper washing and cooking. A broad range of health education program should be launched to vendors and whom who are involved in the growing activities of vegetables and to the general population on the health risks associated with consumption of vegetables being contaminated 
with parasites. The consumers should always obey the fundamental principle of food and personal hygiene that is thorough washing of vegetables before eating and washing hands before meal are recommended.

\section{Acknowledgements}

Authors thanks to the vendors of vegetables for their co-operation during the study and extend their appreciation to the researchers supporting project number (RSP-2020/7) King Saud University, Riyadh, Saudi Arabia.

\section{References}

AL-BINALI, A.M., BELLO, C.S., EL-SHEWY, K. and ABDULLA, S.E., 2006. The prevalence of parasites in commonly used leafy vegetables in South Western, Saudi Arabia. Saudi Medical Journal, vol. 27, no. 5, pp. 613-616. PMid:16680247.

AMOAH, P., DRECHSEL, P., ABAIDOO, R.C. and KLUTSE, A., 2007. Effectiveness of common and improved sanitary washing methods in selected cities of West Africa for the reduction of coliform bacteria and helminth eggs on vegetables. Tropical Medicine E International Health, vol. 12, suppl. 2, pp. 40-50. http://dx.doi.org/10.1111/j.1365-3156.2007.01940.x. PMid: 18005314.

AVCIOǦLU, H., SOYKAN, E. and TARAKCI, U., 2011. Control of helminth contamination of raw vegetables by washing. Vector Borne and Zoonotic Diseases, vol. 11, no. 2, pp. 189-191. http://dx.doi. org/10.1089/vbz.2009.0243. PMid:20569015.

BEKELE, F., TEFERA, T., BIRESAW, G. and YOHANNES, T., 2017. Parasitic contamination of raw vegetables and fruits collected from selected local markets in Arba Minch town, Southern Ethiopia. Infectious Diseases of Poverty, vol. 6, no. 1, pp. 19. http://dx.doi. org/10.1186/s40249-016-0226-6. PMid:28264707.

BENTI, G. and GEMECHU, F., 2014. Parasitic contamination on vegetables irrigated with Awash river in selected farms, eastern Showa, Ethiopia. Journal of Parasitology and Vector Biology, vol. 6, no. 7, pp. 104-110.

BEUCHAT, L.R., 2002. Ecological factors influencing survival and growth of human pathogens on raw fruits and vegetables. Microbes and Infection, vol. 4, no. 4, pp. 413-423. http://dx.doi. org/10.1016/S1286-4579(02)01555-1. PMid:11932192.

DARYANI, A., SHARIF, M., NASROLAHEI, M., KHALILIAN, A., MOHAMMADI, A. and BARZEGAR, G., 2012. Epidemiological survey of the prevalence of intestinal parasites among schoolchildren in Sari, northern Iran. Transactions of the Royal Society of Tropical Medicine and Hygiene, vol. 106, no. 8, pp. 455-459. http://dx.doi.org/10.1016/j.trstmh.2012.05.010. PMid:22703897.

DIAS, M., GEORGE, M. and DIAS, A., 2014. How safe are vegetables: parasitological contamination of fresh leafy vegetables in Manglore. International Journal of Applied Biology, vol. 5, no. 4, pp. 80-82.

DUEDU, K.O., YARNIE, E.A., TETTEH-QUARCOO, P.B., ATTAH, S.K., DONKOR, E.S. and AYEH-KUMI, P.F., 2014. A comparative survey of the prevalence of human parasites found in fresh vegetables sold in supermarkets and open-aired markets in Accra, Ghana. BMC Research Notes, vol. 7, no. 1, pp. 836. http:// dx.doi.org/10.1186/1756-0500-7-836. PMid:25424391.

EBRAHIMZADEH, A., JAMSHIDI, A. and MOHAMMADI, S., 2013. The parasitic contamination of raw vegetables consumed in
Zahedan, Iran. Health Scope, vol. 1, no. 4, pp. 205-209. http:// dx.doi.org/10.17795/jhealthscope-8209.

ELOM, M.O., EZE, U.A., NWORIE, A. and AKPOTOMI, I.O., 2012. Prevalence of geohelminths on edible fruits and vegetables cultivated in. American Journal of Food and Nutrition, vol. 2, no. 3, pp. 58-64. http://dx.doi.org/10.5251/ajfn.2012.2.3.58.64.

ERAKY, M., RASHED, S., NASR, M., EL-HAMSHARY, A. and ELGHANNAM, A., 2014. Parasitic contamination of commonly consumed fresh leafy vegetables in Benha, Egyptian. Journal of Parasitology Research, vol. 25, pp. 617-620. PMid:25024845.

EZATPOUR, B., CHEGENI, A.S., ABDOLLAHPOUR, F., AAZAMI, M. and ALIREZAEI, M., 2013. Prevalence of parasitic contamination of raw vegetables in Khorramabad, Iran. Food Control, vol. 34, no. 1, pp. 92-95. http://dx.doi.org/10.1016/j.foodcont.2013.03.034.

GABRE, R.M. and SHAKIR, A., 2016. Prevalence of some human enteroparasites in commonly consumed raw vegetables in Tabuk, Saudi Arabia. Journal of Food Protection, vol. 79, no. 4, pp.655-658. http://dx.doi.org/10.4315/0362-028X.JFP-15-485. PMid:27052871.

IDAHOSA, O.T., 2011. Parasitic contamination of fresh vegetables sold in Jos markets. Global Journal of Medical Research, vol. 11, no. 1, pp. 21-25.

JAMES, I.M. and OGODUKWUO, A., 2006. A survey of geohelminths ova and larvae on vegetable and fruits sold in Onitsha Southeast Nigeria. Journal of Environmental Health, vol. 3, no. 1, pp. 23-28.

KHAN, W., IMRAN and WAHAB, A., 2016. Intestinal obstruction by Ascaris lumbricoides in a 12 year old boy: a case report in Pakistan. Journal of Bacteriology E Parasitology, vol. 7, no. 01, pp. 262. http://dx.doi.org/10.4172/2155-9597.1000262.

KHAN, W., KHAN, J., KHAN, N., IQBAL, R., ULLAH, A., GHAFFAR, R., MEHMOOD, S.A., AHMAD, S., KHAN, S. and ULLAH, F., 2019b. Soil-transmitted helminth infections in school children of three districts of Malakand region, Khyber Pakhtunkhwa, Pakistan. Pakistan Journal of Pharmaceutical Sciences, vol. 32, no. 2, pp. 799-803. PMid:31103975.

KHAN, W., KHAN, N.I., BUKHARI, S.N.F. and BEGUM, N., 2019a. Prevalence of intestinal parasitic infection among drug addicts in District Swat, Khyber Pakhtunkhwa, Pakistan. Iranian Journal of Parasitology, vol. 14, no. 2, pp. 359-361. PMid:31543928.

KHAN, W., MUMTAZ, G., BIBI, S. and AFZAL, S., 2017c. Parasitic contamination of fresh vegetables sold at upper and lower Dir Districts, Khyber Pakhtunkhwa, Pakistan. Pakistan Journal of Zoology, vol. 49, no. 3, pp. 1115-1118. http://dx.doi.org/10.17582/ journal.pjz/2017.49.3.sc3.

KHAN, W., NOOR-UN-NISA and KHAN, A., 2011. An investigation of incidence of intestinal parasites in under and above 15 years age in farmers of Swat, Pakistan. Proceedings of Parasitology, vol. 52, pp. 43-53.

KHAN, W., NOOR-UN-NISA and KHAN, A., 2012. Endemicity of intestinal parasites with special reference to nematodes in individuals related to education (students, staff and teachers) in Swat, K.P.K, Pakistan. Pakistan Journal of Nematology, vol. 30, no. 1, pp. 77.

KHAN, W., NOOR-UN-NISA and KHAN, A., 2014. A case of Fasciola hepatica infection in Swat, Pakistan. Pakistan Journal of Zoology, vol. 46, no. 6, pp. 1789-1790.

KHAN, W., NOOR-UN-NISA and KHAN, A., 2015. Diversity of intestinal parasites in male and female students and workers of education Department of Swat, Pakistan. Pakistan Journal of Zoology, vol. 47, no. 2, pp. 565-568.

KHAN, W., NOOR-UN-NISA and KHAN, A., 2017a. Soil transmitted helminthiasis in different occupational groups in Swat, Khyber 
Pakhtunkhwa, Pakistan. Pakistan Journal of Pharmaceutical Sciences, vol. 30, no. 4, pp. 1345. PMid:29039336.

KHAN, W., NOOR-UN-NISA and KHAN, A., 2017b. Prevalence and risk factors associated with intestinal parasitic infections among food handlers of Swat, Khyber Pakhtunkhwa, Pakistan.Journal of Food and Nutrition Research, vol. 5, no. 5, pp. 331-336. http:// dx.doi.org/10.12691/jfnr-5-5-7.

KHAN, W., NOOR-UN-NISA and KHAN, A., 2018b. Prevalence of potentially important intestinal pathogenic protozoan parasitic infections in different occupational groups of Swat, Pakistan. Pakistan Journal of Zoology, vol. 50, no. 1. http://dx.doi. org/10.17582/journal.pjz/2018.50.1.123.129.

KHAN, W., NOOR-UN-NISA and NAWAZ, M.A., 2018a. Incidence of tapeworm infection in human population of Swat, Pakistan: an occupation based study. Pakistan Journal of Zoology, vol. 50, no. 2, pp. 639-645. http://dx.doi.org/10.17582/journal. pjz/2018.50.2.639.645.

KHAN, W., ULLAH, A., AHMAD, S. and INAM, Y., 2020a. Helminth Parasites of Zoonotic Importance in Dog Faeces of NorthWestern Region of Pakistan: An Environmental Threat to Public Health. Iranian Journal of Public Health, vol. 49, no. 5 , pp. 1008-1009. http://dx.doi.org/10.18502/ijph.v49i5.3223. PMid:32953693.

KHAN, W., IQBAL, M. and DAD, O., 2020b. Have We Forgotten the Threat Posed by Fascioliasis? A Potential Threat to Public Health. Iranian Journal of Public Health, vol. 49, no. 4, pp. 814 815. PMid:32548067.

LIU, R.H., 2003. Health benefits of fruit and vegetables are from additive and synergistic combinations of phytochemicals. The American Journal of Clinical Nutrition, vol. 78, no. 3, suppl., pp. 517S-520S. http://dx.doi.org/10.1093/ajcn/78.3.517S. PMid:12936943.

LYABO, U.B. and OLUCHI, U.R., 2015. Geohelminth contamination of common fruits and vegetables in Ebonyi State, Nigeria: the public health implication. Bioscience, vol. 1, no. 2, pp. 15-19.

MAIKAI, B.V., ELISHA, I.A. and BABA-ONOJA, E.B.T., 2012. Contamination of vegetables sold in markets with helminth eggs in Zaria metropolis, Kaduna State, Nigeria. Food Control, vol. 28, no. 2, pp. 345-348. http://dx.doi.org/10.1016/j. foodcont.2012.05.035

MAQBOOL, A., KHAN, U.J., YASMIN, G. and SULTANA, R., 2014. Parasitic contamination of vegetables eaten raw in Lahore. Pakistan Journal of Zoology, vol. 46, no. 5, pp. 217-223.

MEHRNEJAT, N., KADKHODAIE, S., FARROKHZADEH, H., YOUSEFI, H.A., POURGHEYSARI, H. and SEYF, S., 2015. Evaluation of parasitic contamination in consuming vegetables in a city of Iran in 2011. International Journal of Environmental Health Engineering, vol. 4, no. 1, pp. 25.

MOHAMED, M.A., SIDDIG, E.E., ELAAGIP, A.H., EDRIS, A.M.M. and NASR, A.A., 2016. Parasitic contamination of fresh vegetables sold at central markets in Khartoum state, Sudan. Annals of Clinical Microbiology and Antimicrobials, vol. 15, no. 1, pp. 17. http://dx.doi.org/10.1186/s12941-016-0133-5. PMid:26968696.

MUNISWAMAPPA, K., RAO, S.D. and VENKATRAMANA, K., 2012. Prevalence of Strongyloides stercoralis contaminating Coriandrum obtained from vendors in Karimnagar. Annals of Tropical Medicine and Public Health, vol. 5, no. 4, pp. 298. http://dx.doi. org/10.4103/1755-6783.102030.

NOOR-UN-NISA, KHAN, W. and KHAN, A., 2012. A Prevalence of intestinal parasites in male and female shepherds of Swat, Pakistan. International Journal of Biology and Biotechnology, vol. 8, no. 4, pp. 597-603.

OGBOLU, D.O., ALLI, O.A., OGUNLEYE, V.F., OLUSOGA-OGBOLU, F.F. and OLAOSUN, I., 2009. The presence of intestinal parasites in selected vegetables from open markets in south western Nigeria. African Journal of Medicine and Medical Sciences, vol. 38, no. 4, pp. 319-324. PMid:20499624.

OMOWAYE, O.S. and AUDU, P.A., 2012. Parasites contamination and distribution on fruits and vegetables in Kogi, Nigeria. Cibtech Journal of Bio-Protocols, vol. 1, no. 1, pp. 44-47.

SIYADATPANAH, A., TABATABAEI, F., EMAMI ZEYDI, A., SPOTIN, A., FALLAH-OMRANI, V., ASSADI, M., MORADI, S., ROSTAMI, A., MEMARI, F. and HAJIALIANI, F., 2013. Parasitic contamination of raw vegetables in Amol, North of Iran. Archives of Clinical Infectious Diseases, vol. 8, no. 2, pp. 1-4. http://dx.doi.org/10.5812/archcid.15983.

SLIFKO, T.R., SMITH, H.V. and ROSE, J.B., 2000. Emerging parasite zoonoses associated with water and food. International Journal for Parasitology, vol. 30, no. 12-13, pp. 1379-1393. http://dx.doi. org/10.1016/S0020-7519(00)00128-4. PMid:11113263.

TEFERA, T., BIRUKSEW, A., MEKONNEN, Z. and ESHETU, T., 2014. Parasitic contamination of fruits and vegetables collected from selected local markets of Jimma Town, Southwest Ethiopia. International Scholarly Research Notices, vol. 2014, 382715. http:// dx.doi.org/10.1155/2014/382715. PMid:27355069.

VALIPOUR, N. R., 2015. Detection of Parasitic Contamination in Ready to Eat Fresh Packaged Herbs Sold in Tehran, Iran.Journal of Community Health Research, vol. 4, no. 2, pp. 99-104.

WALKED, H.M., 2009. Parasitic contamination of vegetables. Journal of Applied Sciences Research, vol. 5, pp. 293-296.

WORLD HEALTH ORGANIZATION - WHO, 1987. Public health significance of intestinal parasitic infections. Bulletin of the World Health Organization, vol. 65, no. 5, pp. 575-588. PMid:3501340. 